\title{
NUEVOS MODELOS DE GESTIÓN LOCAL: LA ARTICULACIÓN DE POLÍTICAS PÚBLICAS Y EL PROTAGONISMO CIUDADANO A TRAVÉS DE LA IMPLEMENTACIÓN DEL PRESUPUESTO PARTICIPATIVO EN LA ARGENTINA
}

\author{
Alejandro López Accotto \\ alopez@ungs.edu.ar \\ Carlos R. Martinez \\ cmartinez@ungs.edu.ar \\ Irene Grinberg \\ igrinberg@ungs.edu.ar \\ Cristian Adaro \\ cadaro@ungs.edu.ar \\ Universidad Nacional de General Sarmiento, Argentina
}

\section{RESUMEN}

El artículo presenta y examina una nueva herramienta en el campo de las políticas públicas: el «presupuesto participativo». El análisis se realiza a partir de una doble mirada: el presupuesto participativo como instrumento de gestión municipal, y como experiencia de participación ciudadana, constituyendo de esta manera un novedoso modelo de integración, organización y gestión local. El estudio se centra en el proceso de implementación del PP en Argentina, explorando las diversas modalidades y criterios de ejecución que ha asumido en los municipios, analizando la articulación con otros actores sociales que intervienen en el proceso, y planteando los avances así como los desafíos que aún quedan por resolver en el perfeccionamiento de un proceso democratizador de las decisiones y las acciones entre gobierno y sociedad.

Palabras clave: presupuesto participativo, democracia participativa, gobiernos locales, gobernanza. 


\section{ABSTRACT}

This article presents and examines a new tool in the territory of public policies: Participatory Budgeting. The analysis is carried out through a double lens: Participatory Budgeting as an instrument of municipal management and as an experience of citizen participation. It constitutes an innovative model of local integration, organization and management. The study focuses on the process of implementing PB in Argentina, exploring diverse execution modalities and criteria that have been applied to municipalities, analyzing the link with other social actors engaged in the process, and setting forth the advances as well as the challenges that remain to be surmounted in perfecting a democratizing process regarding decisions and actions between the government and society.

Key words: participatory budgeting, participatory democracy, local governments, governance. 


\section{INTRODUCCIÓN}

La tendencia a la descentralización y transferencia de competencias desde el nivel nacional hacia los gobiernos locales fue una de las principales medidas que se implementaron durante la década de 1990 como parte de una estrategia de achicamiento y debilitamiento de los gobiernos centrales, a la vez que significaba una minimización del gasto público. Sin embargo, esta política tuvo una connotación dual a nivel local.

Por un lado, los municipios vieron transformarse sus funciones, responsabilidades y atribuciones sin contar con los recursos técnicos, jurídicos, económicos, humanos y administrativos que requería tal incremento en el volumen y complejidad de sus tareas. Por otra parte, se resignificó el ámbito local: los municipios comenzaron a tener una mayor importancia por la cercanía entre gobernantes y gobernados, dando origen entonces a prácticas y formas organizativas muy heterogéneas para hacer frente a la retirada del Estado y especialmente del Estado nacional.

Este escenario creó las condiciones para el surgimiento de la cuestión del desarrollo local $^{1}$ tanto en las agendas ${ }^{2}$ de gobierno como en el ámbito académico, y favoreció una creciente participación ciudadana. En este contexto han ido surgiendo nuevas herramientas de gestión pública vinculadas con estrategias participativas. Los espacios locales, por su escala y cercanía, son un ámbito privilegiado para generar esta participación. Por ello, lograr una verdadera gobernabilidad democrática local es un reto de la nueva gestión municipal orientada al desarrollo sostenible.

El presente artículo toma por objeto de estudio una de las más innovadoras herramientas de lo que se ha llamado la nueva gestión local $^{3}$, que ha surgido en el marco de los cambios sociales, económicos y políticos antes mencionados: el «presupuesto participativo» (en adelante PP). Para ello, se examina su origen, diseño e implementación en Argentina, situando la mirada en dos planos de análisis: su capacidad transformadora como herramienta de gestión local y su potencial como experiencia de democracia participativa. También se analiza el juego de apoyos y 
alianzas entre los gobiernos locales y otras instituciones y actores sociales en el desarrollo de esta importante política pública de participación ciudadana y profundización democrática. Además de la literatura relacionada con la cuestión y la normativa sobre la materia, la principal fuente de información utilizada en el presente estudio son los datos de la Encuesta Permanente a Municipios con PP en Argentina (en adelante EPMPPA) que nuestro equipo del Área de Estado, Gobierno y Administración Pública de la Universidad Nacional de General Sarmiento (en adelante UNGS) viene desarrollando desde el año 2008.

\section{GOBIERNOS LOCALES Y GOBERNANZA}

La República Argentina cuenta con tres niveles de gobierno: el nacional, el provincial y el municipal. Este último se ha configurado en las últimas décadas como el espacio de mayores transformaciones e innovaciones, al menos en lo que se refiere a promover la participación ciudadana en la implementación de políticas públicas.

Tras la ola neoliberal y el paradigma de descentralización de funciones estatales, los gobiernos municipales han pasado a atender demandas de la ciudadanía, crecientes en volumen y diversidad. ${ }^{4}$ Se plantean entonces nuevos desafíos en lo económico, lo político y lo social, que los obliga a repensar, tanto su misión y organización, como sus funciones, las que se han ido desplazando desde el modelo tradicional de administración de las ciudades simbolizado en la tasa municipal de alumbrado, barrido y limpieza como fuente primordial de recursos y declaración de intenciones, a un modelo de gestión en red representativo de un cúmulo de nuevas esferas de acción que implican ahora intervenir en una serie de temas que van desde lo ambiental hasta el desarrollo productivo y el combate a la pobreza, pasando por problemas tales como la atención de la salud, la educación, el cuidado y la seguridad ciudadana.

Las transformaciones e innovaciones de la gestión pública local van en la dirección de reconocer que el Estado ha dejado de tener el monopolio sobre los conocimientos y sobre los recursos económicos e instituciones necesarios para gobernar. Como señalan 
Blanco y Gomá:

las dificultades del Estado ante las transformaciones se asientan a lo largo de cuatro ejes muy dinámicos: las crecientes dificultades del conocimiento, dado el intrínseco carácter incierto de la sociedad post-industrial, y el alto grado de fragmentación cognitiva que ésta implica; la complejidad de valores intereses y preferencias en juego, que no solo poseen múltiples sujetos, sino que son ellos mismos cambiantes e inestables; el carácter insostenible de la concepción jerárquica del gobierno; la creciente interdependencia de problemas y actores políticos, que cuestiona seriamente el modelo clásico de políticas públicas unidireccional y segmentado (2003: 1).

Para explicar y entender tales transformaciones en la relación entre Estado y sociedad, y dar cuenta de las nuevas formas de gobierno que en ellas se originan, la ciencia política creó el concepto de «gobernanza» como una aproximación analítica a estas redes de flujos emergentes. Así, en términos generales, la gobernanza «refiere a un cambio en el equilibrio entre el Estado y la sociedad civil, en el que se pone el acento en la ciudadanía activa y la vincula, en definitiva, a debates más amplios en torno a la democracia deliberativa, el comunitarismo y la visión neorrepublicana de la sociedad civil» (Natera 2004: 10).

Por su parte, el Programa de Naciones Unidas para el Desarrollo, en su aproximación al concepto de gobernanza, explicita que la misma tiene como fin que: «Las personas participen en la toma de decisiones que afectan a sus vidas» (PNUD 2002: 51).

El concepto de gobernanza se encuentra entonces íntimamente relacionado a un incremento en la densidad de la democracia, por medio de la combinación de los mecanismos tradicionales de representación política con otros de tipo más directo vinculados con la motorización de intereses y demandas de tipo sectorial o comunitario. Se puede afirmar que la gobernanza alude a un nuevo estilo de gobierno y gestión pública caracterizado fundamentalmente por un mayor grado de cooperación y vinculación entre el Estado y actores no estatales en el marco de redes decisionales que 
incluyen a los diferentes niveles de gobierno, al sector privado y a distintos actores sin afán de lucro. ${ }^{5}$ De este modo, es posible identificar dos puntos de vista en el concepto de gobernanza:

La versión negativa resalta la capacidad de las fuerzas sociales para resistir las imposiciones y regulaciones de la administración. Aquí, el elemento normativo es que los ciudadanos saben mejor que el Estado lo que quieren y necesitan y, por tanto, está perfectamente justificado que intenten encontrar la forma de evitar las incursiones de la autoridad y la burocracia en su esfera. La versión positiva, por su parte, apuntaría a que existen recursos en la sociedad lo suficientemente sólidos como para poder diseñar e implementar eficazmente las políticas y esos recursos deben ser aprovechados. Se asume que las redes, comunidades u otros agregados de organizaciones de intereses existentes en los distintos sectores de políticas están en disposición de dar forma a las políticas o asistir al gobierno en su implementación. La fuerza e incluso la dominación de la sociedad constituyen una ventaja para el gobierno, pues permite al sistema llegar a decisiones mejores (en el sentido de más eficaces, eficientes y justas) (Navarro 2002: 2).

El presente trabajo parte de la segunda visión, identificada con el republicanismo y la democracia, y no de la primera, más cercana al liberalismo. ${ }^{6}$ No se trata entonces de cuestionar la acción pública, identificando la gobernanza con la resistencia de la sociedad ante el avance del Estado, sino de mostrar las potencialidades de la acción concertada y cooperativa de actores y recursos estatales y no estatales en pos de lograr una mejora en el funcionamiento y los resultados de las políticas públicas. De esta forma, la noción básica que identificamos a partir del concepto de gobernanza es la decisión política de las autoridades estatales, más o menos voluntaria, de compartir el poder y de democratizar las decisiones de gobierno incorporando a actores no estatales, que se espera representen lo más ampliamente posible los distintos intereses sociales en el diseño, implementación, evaluación y control de las acciones del gobierno.

En cuanto al ámbito local, la riqueza de la gobernanza y de la gestión de redes de participación ciudadana radican en «la identificación de la organización municipal con 
las peculiaridades y los intereses del colectivo y del territorio al que atiende; visibilidad, cercanía y accesibilidad; la idea de que la administración local es más versátil y flexible para atender las demandas concretas de los ciudadanos; las mayores posibilidades de las administraciones locales de crear las condiciones favorables para la inclusión de individuos o colectivos interesados en los temas públicos» (Natera 2004: 23).

De esta forma, es clara la ventaja comparativa del nivel local de gobierno para transformar la gestión pública desde un modelo tradicional hacia uno participativo frente a los gobiernos centrales y provinciales, dado que es la forma más cotidiana, accesible y conocida de Estado para buena parte de la población. Entonces, los riesgos de constituir la primera línea de contención de las demandas sociales pueden volverse una oportunidad para el tránsito hacia la construcción de un Estado mejor, más eficaz, justo y transparente. Así, puede afirmarse que: «Las redes en el nivel local tienen una característica que las diferencia de las que se constituyen a nivel nacional y es que tienden a incorporar en su seno a los ciudadanos anónimos. Mientras que la noción de gobernanza en el nivel de gobierno central nos hace pensar en redes constituidas por actores públicos (correspondientes a los distintos niveles de gobierno —incluido el nivel supranacional- y demás agencias públicas) y por representantes de grupos económicos y sociales (mundo empresarial, ONGs, asociaciones, etc.), en las formas locales de articulación de las decisiones encontraríamos con relativa frecuencia a los ciudadanos como actores de la red, esto es, presentes en foros, grupos y otras fórmulas relacionales que aseguran su participación en los procesos decisorios. La participación ciudadana en el ámbito local y no en otros es hasta cierto punto lógica si consideramos que éste es el nivel de gobierno más próximo al ciudadano y donde se puede articular de una manera más efectiva» (Navarro 2002: 6).

En conclusión, la gobernanza puede oficiar de hilo conductor entre las teorías de la nueva gestión pública, sobre todo a nivel municipal, y el concepto de desarrollo local.

Ello es posible si se define el desarrollo local como algo más que el mero crecimiento o 
aún diversificación de la economía, contemplando asimismo una efectiva mejora en las condiciones de vida de la población, una mayor justicia distributiva, la sustentabilidad en materia medioambiental y la profundización de la vida democrática. Si además se identifica a los nuevos modelos de gestión local como más abiertos, participativos y transparentes respecto a la concepción tradicional de la administración pública municipal, así podríamos conceptualizar la gobernanza y los distintos instrumentos utilizados para fortalecerla, entre los que se inscribe el PP, como los cambios en el desarrollo de las políticas públicas municipales necesarios para lograr un modelo de gestión participativo que permita conducir y fortalecer los procesos multidimensionales de desarrollo local.

\section{EL PRESUPUESTO PARTICIPATIVO Y SU EVOLUCIÓN EN LA ARGENTINA}

El PP, herramienta de participación popular, combina las democracias representativa (indirecta) y participativa (directa). Así, sería: «una forma de gobierno público que intenta romper con la tradición autoritaria y paternalista de las políticas públicas, recurriendo a la participación de la población en diferentes etapas de la preparación e implementación presupuestaria, con un énfasis especial en la definición de prioridades para la distribución de las recursos de inversión» (Santos 2004: 25).

La visión original presente en la ciudad donde nace el PP, la brasileña Porto Alegre, parte de un diagnóstico negativo de las políticas públicas identificadas con la tradición paternalista y autoritaria, vigente en buena parte de América Latina, pero no busca reducir la esfera de lo público sino revalorizarla con la participación popular. Así, uno de sus impulsores en Porto Alegre lo definió como: «un proceso de democracia directa, voluntaria y universal, donde el pueblo puede discutir y decidir sobre el presupuesto y las políticas públicas. El ciudadano no limita su participación al acto de votar para elegir al Ejecutivo o al Parlamento, sino que también decide las prioridades de gastos y controla la gestión de gobierno. Deja de ser un coadyuvante de la política tradicional para ser protagonista permanente de la gestión pública» (Genro y Sousa 1998: 123). 
En esta concepción, es posible advertir la idea de que el PP permite fortalecer la democracia, no negando la importancia de su faz delegativa (que en buena parte de América Latina ha sido un derecho que, merced a la participación popular, se ha arrancado de las garras de feroces dictaduras) sino enriqueciéndola con mecanismos participativos. Algunos de los objetivos que el PP puede contribuir a lograr, en tanto instrumento político y por tanto herramienta de transformación de la realidad, son:

a) El cambio de las condiciones de vida y mejoras de infraestructura en toda la ciudad, manteniendo una propuesta equilibradora en lo territorial y distributiva en lo económico.

b) La recuperación del prestigio social de la política.

c) La participación ciudadana.

d) La inversión de prioridades al favorecer o discriminar positivamente a los grupos o sectores más vulnerables.

e) La ruptura del clientelismo.

f) El perfeccionamiento de la gestión local.

g) El control fiscal por parte de la ciudadanía al Estado.

h) La combinación de democracia participativa y directa.

i) La democratización de las relaciones del Estado con la sociedad y la creación de una esfera pública, no estatal. ${ }^{7}$

Desde su nacimiento en 1989 en Porto Alegre, el PP atravesó tres fases históricas: «Fase I: Experimentaciones, desde 1989 hasta 1997, Fase II: Masificación brasileña de 1997 a 2000, y Fase III: Expansión fuera de Brasil y diversificación, a partir de 2000» (Cabannes 2005: 8). A esta última corresponde su implementación en Argentina.

En la República Argentina, la aplicación del PP surge tras el estallido económico, político y social de fines de 2001, en el marco de una profunda crisis de representación. A lo largo de la primera década del siglo XXI, un creciente número de gobiernos locales ha comenzado a aplicar el PP, sumando un total de cuarenta y un municipios en 2010. 
La importancia poblacional de dichos territorios es tal que, en 2010, uno de cada cuatro habitantes de la nación vivía en un municipio con PP (once millones de personas).

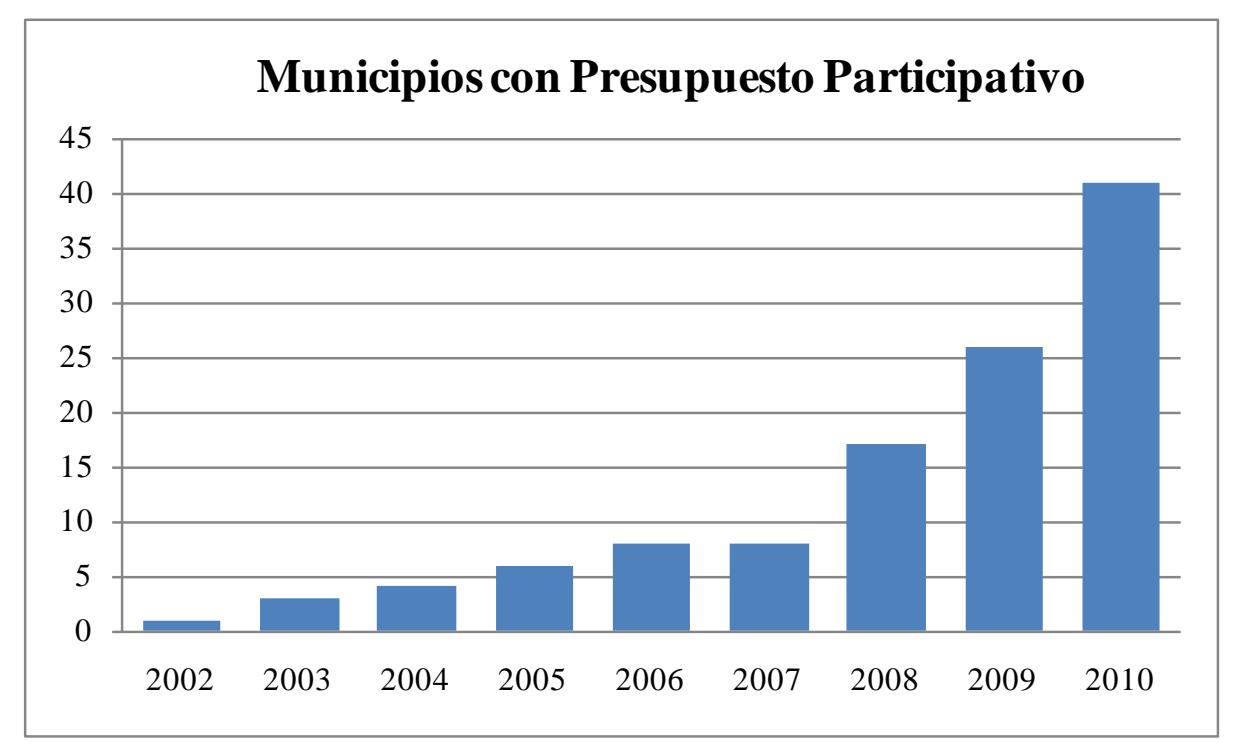

Fuente: Red Argentina de Presupuestos Participativos, en www.rapp.gov.ar

En cuanto a su distribución territorial, la mayor parte de los municipios con PP en Argentina se concentra en las provincias más pobladas y desarrolladas: Buenos Aires (14), y en menor medida Santa Fe (7), Córdoba (4) y Mendoza (3). Destaca Tierra del Fuego, donde sus dos municipios tienen PP. 


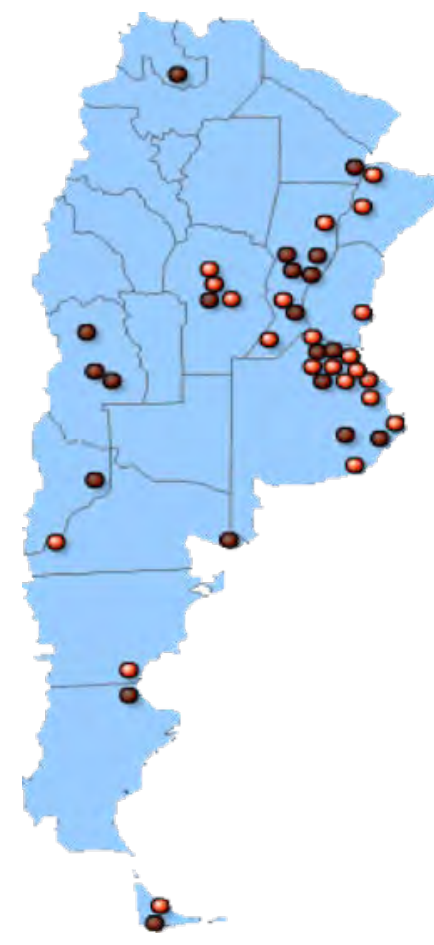

Fuente: Elaboración propia

Si bien no puede deducirse de los datos anteriormente citados una relación unívoca y directa entre grado de desarrollo y adopción de la herramienta, pocos considerarían casual que el PP casi no haya sido implantado en las regiones que presentan un mayor atraso relativo en términos de desarrollo.

\section{MARCO INSTITUCIONAL DEL PRESUPUESTO PARTICIPATIVO EN ARGENTINA}

En Argentina, el art. $1^{\circ}$ de la Constitución Nacional (CN) establece un sistema de gobierno que adopta la «forma representativa, republicana y federal». A su vez, el art. 22 de la CN define cabalmente la forma representativa al normar que: «El pueblo no delibera ni gobierna, sino por medio de sus representantes y autoridades creadas por esta Constitución». Sin embargo, la misma CN habilita algunos mecanismos de democracia semi-directa, como el de iniciativa popular (art. 39), que permite a la ciudadanía la presentación de proyectos de ley, y el de consulta popular (art. 43), por el cual el voto directo de la población permite transformar proyectos en leyes. 
Por su parte, el artículo 75, inciso 22, de la $\mathrm{CN}$, establece que una serie de declaraciones, convenciones y pactos internacionales suscritos por el Poder Ejecutivo nacional, y aprobados por el Congreso de la Nación, tienen jerarquía constitucional, así como las condiciones necesarias para que eventualmente otros tratados se agreguen a tal listado, declarándolos complementarios de los derechos y garantías consagrados en la CN. En tal sentido, dos artículos de tratados internacionales habilitan la participación directa de la ciudadanía: el artículo 21 de la Declaración Universal de Derechos Humanos y el artículo 25 del Pacto Internacional de Derechos Civiles y Políticos, que garantizan el derecho a la participación, directa o indirecta, en los asuntos públicos, a todas las personas. El derecho a la participación, directa e indirecta, en los asuntos de Estado, está garantizado entonces por la Carta Magna de la nación y por pactos internacionales con rango constitucional

En el segundo nivel del Estado, varias provincias promueven la aplicación del PP. El régimen municipal de la provincia de Entre Ríos, fundamentado en la Ley provincial n. 3,001 actualizada en 2006, establece en su artículo 120 respecto al presupuesto municipal que los municipios pueden adoptar el PP y que en todas las etapas de su proceso pueden participar los ciudadanos sin otra restricción que la mayoría de edad, mencionando específicamente el rol del control ciudadano sobre la gestión pública. Sin soslayar lo valioso de la norma, es necesario recordar que, sin embargo, en Entre Ríos sólo un $10 \%$ de los municipios tienen PP.

También en el nivel subnacional, la Ciudad Autónoma de Buenos Aires, que tiene un status de cuasi-provincia, en su Constitución reconoce el presupuesto participativo en su artículo 52, el cual establece el carácter participativo del presupuesto. Finalmente, la provincia de Buenos Aires instrumentó el Decreto N $\mathrm{N}^{\mathrm{3}} 3.333 / 05$ y creó el Programa Provincial para la Implementación Progresiva del Presupuesto Participativo que, entre otras cuestiones, invita a los municipios a sumarse a esta iniciativa. Si bien el programa no se desarrolló cabalmente, el mencionado decreto suele ser utilizado en los antecedentes y justificaciones de proyectos de ordenanzas en los municipios 
bonaerenses a la hora de establecer el PP.

En el último de los niveles del Estado argentino, el local, encontramos la contribución más significativa a la instalación del PP, tanto por su incorporación en las cartas orgánicas, como en la sanción de ordenanzas, decretos u otras normas municipales. Así, en algunas provincias donde la autonomía municipal permite a los Estados locales tener su propia carta orgánica, el PP está contemplado en la misma. Como ejemplo se pueden mencionar las cartas orgánicas de los municipios de Posadas, capital de la provincia de Misiones, y de Resistencia, capital de la provincia del Chaco, ambas en el noreste del país, que contemplan explícitamente el PP. En el caso de Posadas, el mismo no se ha implementado mientras que Resistencia desarrolla el PP desde el año 2009.

Resulta importante señalar que, si bien el marco institucional obliga a la aplicación del PP o lo habilita, según los casos, en los hechos no es determinante, dado que muchas veces la política se implementa a pesar de no contar con un gran respaldo normativo. Sin embargo, en otras experiencias, a pesar de lo establecido en la legislación provincial y aún en las mismas normas del municipio, el PP no se lleva a la práctica. Se verifica así que, más allá de ciertos avances en el terreno legislativo y normativo, en los hechos la proliferación de experiencias de $\mathrm{PP}$ en Argentina en los últimos años se explica principalmente por fuertes decisiones políticas del poder ejecutivo local en los distritos donde las mismas han tenido lugar, y por cierta presencia del tema en la agenda del Poder Ejecutivo nacional, materializada fundamentalmente en acciones concretas de promoción de la herramienta tales como la capacitación y el aprendizaje horizontal entre municipios con diferentes grados de experiencia en PP.

\section{EL PRESUPUESTO PARTICIPATIVO: DISTRIBUCIÓN Y REGLAMENTO}

Las experiencias de PP en los municipios argentinos presentan una gran variedad de criterios a la hora de distribuir los recursos y de reglamentar las distintas etapas que conforman el proceso. Un primer punto consiste en identificar quién decide acerca de la distribución de los recursos que integran el PP, lo que implica una cuestión más amplia: 
dar cuenta de qué actor o actores reglamentan en forma general el proceso.

En Argentina, las distintas experiencias de PP han sido iniciativas del poder político local. Según la EPMPPA, en un $40 \%$ de los municipios el PP surge, al menos normativamente, como una ordenanza del concejo deliberante, esto es, del poder legislativo local. Tal tipo de norma suele tener márgenes más o menos amplios para su posterior reglamentación por parte del poder ejecutivo local. Así, en la totalidad de los casos conocidos, el reglamento del PP, al menos durante el primer año, emana de la autoridad política. Ello no quita que en algunas experiencias haya surgido con el correr del tiempo un reglamento pactado por los propios vecinos, que incluye, en varios casos, las formas y criterios de distribución de los fondos del PP. Sin embargo, subsiste un tema que, al menos en Argentina, queda siempre como una potestad del gobierno local, la determinación del monto total o porcentaje del presupuesto municipal general que se pone a consideración de la ciudadanía. A partir de datos de la EPMPPA, se observa que lo habitual es que los municipios con PP en Argentina destinen al mismo entre un 1\% y un $2 \%$ de sus recursos totales, con valores mínimos y máximos registrados del orden del $0,2 \%$ y del 7\%, respectivamente. Vale aclarar, por si los montos parecen exiguos, que por lo general el PP opera con una lógica de base cero, ${ }^{8}$ es decir, que cada año la ciudadanía discute nuevamente el monto total a poner en juego, lo que imposibilita tratar proyectos de gran envergadura, con carácter plurianual, aunque no quita que algunas iniciativas se repitan año a año. Tampoco se consideran en el PP gastos recurrentes, esto es erogaciones de carácter permanente como salarios u otro tipo de pagos continuos por bienes, servicios o transferencias. Por tanto, el porcentaje asignado al PP depende, en parte, del grado de rigidez de los respectivos presupuestos municipales. Así, los gobiernos locales pueden asignar al PP los recursos que exceden sus gastos en personal, servicio de la deuda y servicios especiales urbanos (recolección y disposición final de residuos sólidos), entre otros pagos perentorios. Por lo anterior, el margen de asignación de recursos al PP depende de variables históricas y estructurales que condicionan la voluntad política de la administración local. 
Una vez dispuesto el monto de recursos que ha de tratarse en el PP, la siguiente cuestión es determinar la base conceptual a partir de la cual se distribuirán tales recursos públicos. Ello puede formar parte de la autoreglamentación aunque, al igual que otras decisiones sobre distribución de fondos, por lo general no se incorpora al debate en el primer año, no por menospreciar el potencial de los acuerdos populares, sino para facilitar el proceso sin introducir desde el principio una discusión que puede empantanar los debates, especialmente cuando no se ha desarrollado plenamente una cultura participativa entre la población. Las modalidades que suele adoptar conceptualmente la distribución de recursos en el marco del PP son de dos tipos. La más habitual, predominante en Argentina, consiste en distribuir los recursos con algún criterio territorial, esto es, a partir de una subdivisión geográfica del municipio. La otra modalidad, menos difundida pero de gran riqueza conceptual y que puede yuxtaponerse a la anterior, es el desarrollo de ejes temáticos, reconociendo que existen cuestiones, como el transporte, que incumben a varias regiones del municipio y que no pueden acometerse a través de un enfoque parcelado. También pueden destinarse recursos en forma separada para atender la problemática especial de determinados grupos poblacionales, como pueden ser los y las jóvenes o los niños y niñas, reconociendo necesidades y visiones particulares y distintas en tales generaciones respecto a las del conjunto indiferenciado de la población. En Argentina prima el uso de una distribución territorial, si bien cada vez más municipios cuentan con PP joven e incluso algunos evalúan introducir un PP destinado a la niñez.

Es interesante señalar la relación entre PP y descentralización municipal. En algunos casos, como el del municipio de Morón (del área metropolitana de Buenos Aires), el PP se distribuye geográficamente de acuerdo a la subdivisión del territorio que previamente se había realizado en el marco de un proceso de descentralización municipal dirigido a acercar y facilitar a la población el acceso a los servicios y trámites municipales. Así, el PP estuvo diseñado para apoyarse en un proceso previo de descentralización. Simétricamente, en el municipio de Villa María (de la zona central del país) la distribución espacial realizada en el marco del PP se usó para la descentralización 
municipal, que a su vez se apoyó en el PP para obtener visibilidad en el territorio. Cerca del $40 \%$ de los municipios considerados en la EPMPPA declaran que el PP se vincula con un proceso de descentralización municipal.

En cuanto a los criterios de distribución geográfica de los recursos al interior del territorio, es habitual que, en una primera etapa, sean definidos por los municipios sin ponerse a discusión entre la población. En casi el 30\% de las experiencias captadas por la EPMPPA se ha introducido algún criterio de distribución según necesidades aplicando indicadores objetivos, como por ejemplo el de necesidades básicas insatisfechas, o información propia sobre carencia de equipamiento urbano u otras necesidades. Tal tipo de criterio es a su vez coherente con los que surgen en los casos donde la propia población discute la distribución. Por ejemplo, en el municipio de Zárate, en la provincia de Buenos Aires, en su autoreglamento vigente a partir del segundo año de desarrollo del PP, los participantes establecieron la necesidad de considerar criterios de justicia social en el reparto de los fondos. De este modo se verifica en la práctica que el PP suele contemplar, al menos una vez consolidado como política de Estado a nivel local, un objetivo de redistribución del gasto hacia los sectores sociales y zonas del territorio más postergados.

En cuanto a las dimensiones de los proyectos, un 20\% de los municipios ha informado en la EPMPPA que incorporaron topes al costo máximo de los proyectos. A su vez, un $15 \%$ de los distritos considera que los proyectos deben tener cierta escala para lograr un impacto perceptible en la vida de la población, y por tanto han establecido un número máximo de proyectos a ser financiados por el PP para evitar una fragmentación extrema en las acciones desarrolladas. El 30\% de los municipios ha financiado con el PP no más de diez proyectos cada uno, mientras que otro 30\% de los municipios encuestados ha llevado adelante más de 50 proyectos cada uno.

$\mathrm{Al}$ respecto, interesa señalar que una escala demasiado pequeña en la dimensión de los proyectos conspira contra la posibilidad de que la ciudadanía discuta cuestiones cada vez más importantes y de creciente trascendencia para la vida de la población. Este 
aspecto, junto con el alcance de la autoreglamentación, son cuestiones sustantivas que contribuyen a la calidad de los procesos participativos.

\section{PRESUPUESTO PARTICIPATIVO Y PARTICIPACIÓN CIUDADANA: ALCANCE Y FORMAS}

Vale recordar que el PP, por definición, consiste en la distribución de una parte de los gastos presupuestados en un determinado año a través de la decisión de una parte de la ciudadanía identificada como aquellas personas que habitan un territorio y que tienen la voluntad y disponibilidad de colaborar en el proceso. De ahí que, a la hora de medir su importancia cuantitativa, resulte clave conocer la proporción de la población de cada distrito que forma parte del proceso decisorio desarrollado a través del PP. Dado que cada distrito desarrolla el proceso, y por tanto define y cuantifica la participación de la población de un modo diferente, las comparaciones cuantitativas deben relativizarse.

Una primera cuestión que surge es cómo medir la participación. Por ejemplo: ¿debe considerarse igual a dos personas que asisten a una reunión cada una, que a otra que participa de dos reuniones?, ¿ hay que establecer un número mínimo de presencias para considerar a un mero asistente como un participante real?

Del mismo modo, no son iguales, en términos de la calidad de la participación, las distintas formas de implementación del PP. Así, en algunas experiencias se establecen foros barriales cuyos asistentes, en muchos casos, realizan un diagnóstico, definen prioridades, escogen alternativas e incluso participan en el diseño de los proyectos a ejecutar. En otros casos, la decisión final corre por cuenta de un colectivo más amplio que el de los participantes en los foros. En las experiencias de este tipo, los asambleístas buscan generar apoyo para sus ideas y propuestas en eventos de carácter más masivo, comúnmente denominados ferias de proyectos, a las que suelen asistir ciudadanos que no han tomado parte en ninguna reunión previa.

Es claro que no es equivalente la participación de quien desarrolla todo el proceso a la de quien se limita a participar en la elección final, y aún en este caso resta resolver el 
grado de correspondencia entre quien asiste personalmente a una feria de proyectos y quien vota en forma no presencial a través de Internet, teléfono u otras vías. En resumen, lo que cada comuna considera como participantes está íntimamente ligado al concepto de participación incorporado en el diseño del PP en cada municipio.

A su vez, resulta claro que la base para una participación real es la información previa. Solamente las decisiones informadas pueden considerarse estrictamente tales, sin sombra de duda acerca de manipulaciones sobre las mismas. Por ello, resulta particularmente preocupante el hecho de que un 20\% de los municipios de los que se cuenta con datos no contemple procesos previos de información a la ciudadanía acerca del PP. No es de extrañar entonces que, en estos casos, no solamente la calidad de la participación resulte seriamente comprometida, sino que incluso los niveles de participación logrados en estos distritos sean un $40 \%$ inferiores a los obtenidos por los municipios que sí han desarrollado procesos previos de información ciudadana.

El nivel de participación informado en la EPMPPA es, en promedio, del orden del 1\% de la población total de cada distrito. Sin embargo, en un 40\% de los casos tal guarismo no llega al $0,5 \%$. Este resultado no es de por sí positivo ni negativo, sino que su evaluación depende en buena parte de la calidad de los procesos participativos. Así, la participación de una persona debidamente informada sobre el tema en media docena de asambleas sobre la definición de prioridades, el diseño de proyectos, la elección de alternativas, el control de lo ejecutado y la reglamentación del proceso, podría valorarse como de un aporte mayor que el hecho de que diez habitantes de un territorio hayan votado, presencial o telemáticamente, un proyecto que no terminan de comprender bien por falta de información sobre cómo se ha originado, en qué consiste o en el marco de qué procesos se inscribe.

Otra cuestión que llama la atención es que el 30\% de los municipios de los que se cuenta con datos reconozca una tendencia decreciente en la evolución de la participación. En todos los casos se trata de municipios que informan que el nivel de 
visibilidad y conocimiento acerca de la experiencia de PP entre los habitantes no es buena, sino apenas regular. A ello se suma, en algunos casos, la inexistencia de procesos previos de información a la ciudadanía y, en otros, un mal funcionamiento en los mecanismos de ejecución de las obras y servicios que surgen del PP. Si la cantidad de participantes, absoluta o relativa, poco dice acerca del potencial de cada experiencia para profundizar la vida democrática, la evolución de la misma arroja algunas pistas acerca de la buena salud de los procesos.

Así, los datos disponibles permiten colegir que una caída en la participación podría relacionarse con fallas en la labor del gobierno local tendiente a sostener el PP, bajo la forma de una insuficiente difusión del PP, procesos de información ciudadana débiles, e incluso atrasos y problemas en la ejecución de los proyectos priorizados por la población. Todo ello implica descrédito e indiferencia popular respecto al PP y reduce en gran medida sus posibilidades de sostenerse como política y cumplir con sus objetivos: promover la conciencia ciudadana, los valores comunitarios y la cultura de la participación.

Esta revalorización y reapropiación de lo público funciona como una comunicación de dos vías, no solamente permitiendo una mejor atención de las necesidades de la población, sino también incrementando los recursos públicos que solventan tales servicios. Así, aunque los municipios encuestados no consideran relevante el efecto positivo del PP sobre el cobro de las tasas municipales, la noción de ciudadanía incluye la idea de ciudadanía fiscal entendida como el principio que sostiene que los servicios públicos, es decir de todos y todas, deben ser financiados por la comunidad íntegra. Ello está presente, por ejemplo, en la experiencia del municipio de Mendoza (en el oeste de Argentina y capital de la provincia homónima) donde la política ha sido impulsada desde la Secretaría de Hacienda y la concepción integral de construcción de ciudadanía incluye la ampliación de la ciudadanía fiscal. ${ }^{9}$

Se concluye que, por la heterogeneidad con que se aplica el PP, no existe un solo tipo ni 
una calidad única de participación. El potencial de la participación ciudadana es de por si enorme aún si consideramos únicamente sus efectos directos, producidos por el crecimiento personal, comunitario y como colectivo social, de los vecinos y vecinas que en asamblea deciden por sí mismos el destino de parte de los fondos públicos.

\section{EL PRESUPUESTO PARTICIPATIVO COMO INSTRUMENTO DE GESTIÓN PÚBLICA LOCAL}

Como ya se ha detallado, un primer efecto del PP en términos de impacto sobre la gestión pública local radica en su capacidad para complementarse con procesos de descentralización territorial. Incluso en los municipios en que tales procesos no se han desarrollado, el PP ha servido como una forma de territorializar la gestión en términos de llevar la acción estatal local a todos los rincones del distrito, incluyendo a regiones históricamente postergadas u olvidadas en las agendas públicas de gobierno.

Un segundo efecto, en este caso directo, de la adopción del PP por parte del gobierno local, es la incorporación en su estructura funcional de nuevas actividades en el marco del PP. Al respecto, la categoría que se otorga al área encargada del PP puede ser un indicador de la importancia que se asigna a la cuestión en la agenda política del gobierno. En el caso argentino, la EPMPPA revela que en el 80\% de los casos se trata de una Secretaría, el máximo nivel jerárquico contemplado generalmente en las administraciones públicas locales argentinas. Sin embargo, de la misma encuesta surge que en el $70 \%$ de los casos tales áreas cuentan con menos de diez funcionarios y que la media es de menos de seis funcionarios.

Sin perjuicio de ello, la lógica de funcionamiento del PP hace imprescindible que funcionarios de todas las áreas de gobierno estén implicados con la política a fin de agilizar la ejecución de los proyectos y determinar su factibilidad técnica. Además, un modelo de gestión participativa requiere de un cambio institucional para asegurar su buen funcionamiento. Así, es fundamental que personal de distintas áreas del gobierno municipal (legal, hacienda, obras públicas, salud y cultura, entre otras) participe más o 
menos activamente en el proceso de PP.

De este modo, es posible observar que no todas las personas que trabajan en las áreas del PP realizan una labor directamente vinculada con el proceso, por tratarse en muchos casos de personal de apoyo, y que el número de funcionarios de otras áreas que efectivamente se dedican al PP es bastante acotado. Desde el punto de vista del impacto directo del PP sobre las estructuras funcionales de los municipios argentinos, el resultado es más bien modesto. Ello genera algunas dudas sobre la capacidad de los distintos gobiernos locales para sostener adecuadamente el proceso, dado que el PP, como cualquier otra política pública, necesita de cierta masa crítica en materia de recursos financieros, materiales, organizacionales y humanos para desarrollarse correctamente y lograr los objetivos buscados.

No obstante, el principal efecto que la adopción del PP debería generar sobre el modelo de gestión de los gobiernos locales es de tipo indirecto, ya que no es posible sostener una política de esta naturaleza con una estructura vertical y compartimentada. Esta circunstancia es ampliamente reconocida por los municipios que han implementado el PP, como se constata en el hecho de que, según la EPMPPA, el 90\% de los distritos encuestados considera que la participación de las estructuras técnicas sectoriales es relevante o muy relevante en el desarrollo del PP.

Para garantizar su desarrollo y sostenibilidad, el PP requiere un cambio en la forma de funcionar de los municipios, en tanto la participación popular exige brindar respuestas, literalmente, a los distintos requerimientos de la ciudadanía. Así, es habitual que los responsables técnicos de las distintas áreas del gobierno municipal participen de los foros, al menos en la instancia de información sobre la viabilidad de los distintos proyectos o ideas propuestas por la población. Tal presencia implica a veces el primer contacto entre los responsables de las distintas políticas públicas locales y la ciudadanía a la que están dirigidas, tratándose de un espacio de intercambio mutuo que es valioso tanto para los ciudadanos, que pueden así comprender mejor el funcionamiento del 
gobierno local y plantear sus demandas con mayor efectividad, como para los propios funcionarios, que pueden encontrar en la relación directa con la población un elemento enriquecedor de sus prácticas.

A su vez, el hecho de que en el $85 \%$ de los casos relevados por la EPMPPA se contemplen mecanismos de participación popular en el control de los proyectos, ayuda a la instalación de una cultura de control en las administraciones públicas locales, lo que permite operar con mayor transparencia y efectividad.

En lo referente a los legisladores locales (concejales), en el $60 \%$ de las experiencias encuestadas el PP se origina a partir de una ordenanza votada por el concejo deliberante, organismo que desempeñará un papel relevante en su implementación. En el 100\% de los casos, los legisladores conservan el control ya que los presupuestos locales, incluida la parte de los mismos destinada al PP, deben ser aprobados por el concejo deliberante. Así, los legisladores conservan la potestad de legalizar y hacer efectivo o no el mandato popular vigente en el PP.

De este modo, a nivel político el PP brinda la posibilidad de ganar legitimidad, redoblando la apuesta por más y mejor democracia y por una ciudadanía informada que pueda mejorar y apreciar los resultados de la gestión, oportunidad útil tanto por los representantes del poder ejecutivo como legislativo local. Mientras que a nivel técnico el PP permite instaurar al interior de las administraciones públicas locales un modelo de gestión participativa y una cultura de control, contribuye también a mejorar las capacidades de los funcionarios, quienes en el foro público reconocen las necesidades de la población y son reconocidos por el valor público ${ }^{10}$ que logran generar.

El desarrollo local que el PP promueve parte tanto de una intensificación de la vida democrática, posible por un más pleno ejercicio de los derechos humanos relacionados con las opciones de todas las personas para participar de las decisiones que las afectan, como de un fortalecimiento de la gestión pública, que, lejos de limitarse a los proyectos 
del PP, permite contar con un aparato público mejor preparado para fomentar la actividad económica, mejorar las condiciones de vida de la población y garantizar la sustentabilidad medioambiental, entre otras cuestiones que atañen al desarrollo local.

\section{ARTICULACIÓN DEL PRESUPUESTO PARTICIPATIVO CON OTROS ACTORES SOCIALES}

El PP, como cualquier otra política pública, requiere para su éxito de la concurrencia y cooperación de una red compleja de actores, y se diferencia de otras herramientas participativas y formas de cogobierno por el énfasis puesto en interpelar a los ciudadanos en su carácter de tales, es decir, no como miembros de ningún colectivo particular, más allá del de habitantes de un determinado territorio. Ello no impide que en muchos casos se reserve cierto protagonismo a las distintas organizaciones de la sociedad civil durante el proceso del PP, tal como lo declara un 50\% de los municipios encuestados.

Esta circunstancia se relaciona, en algunos casos, con la historia del territorio y la forma en que el PP se desarrolló. Así, por ejemplo, en el caso de San Miguel (municipio que integra el área metropolitana de la Ciudad de Buenos Aires) el PP se originó como una demanda social promovida por un conjunto de organizaciones de la sociedad civil que lograron imponerla, primero en la agenda legislativa y luego entre las prioridades de gobierno. Tanto el protagónico rol reservado a las organizaciones en el PP, como el hecho de que San Miguel sea el municipio que mayor porcentaje de sus recursos totales (7\%) asigna a este proceso, en buena medida obedecen a esta historia.

Son muchos los casos en que distintas organizaciones ajenas al entramado de actores técnicos y políticos de los poderes legislativos y ejecutivos locales han realizado un aporte significativo al PP en Argentina: un 70\% de los gobiernos municipales encuestados declara que han desarrollado el PP contando con apoyos de organizaciones externas. Se verifica entonces, en línea con la idea de gobernanza, que el desarrollo de las políticas públicas, máxime en el caso de las de carácter participativo, requiere de una 
suma de saberes y de la movilización de una diversidad tal de recursos, que muchas veces superan las posibilidades del actor estatal que la impulsa.

En el 40\% de las respuestas obtenidas por la EPMPPA se señala que las universidades han representado un apoyo significativo para el desarrollo del PP, destacándose su aporte en la dotación de solvencia técnica al proceso. Resulta interesante considerar si el rol de las universidades se ha limitado a atender las solicitudes de asistencia técnica de las comunas, o si además han funcionado como agentes de difusión y promoción del PP haciéndolo visible en las agendas políticas de los gobiernos locales. La idea de que el papel de las universidades en los procesos de PP se relaciona con su vinculación con el territorio se refrenda en el hecho de que se trata de instituciones públicas cuyo radio de acción se identifica con las zonas en que más se ha desarrollado la herramienta, tales como la región nordeste del área metropolitana de la Ciudad de Buenos Aires, la Patagonia o las márgenes del alto Paraná.

A su vez, el 30\% de los apoyos obtenidos por los municipios para desarrollar el PP ha provenido de una pléyade de actores estatales, tales como otros municipios con experiencia en la materia, estados provinciales, el Estado nacional y agencias de cooperación internacional, incluyendo a organizaciones vinculadas a las Naciones Unidas. En este caso se ve nuevamente la potencialidad, en términos de sinergias y aprendizajes cruzados, de la instauración de foros de intercambio de experiencias acerca del desarrollo del PP en Argentina.

En cuanto a los apoyos de organizaciones de la sociedad civil, según las encuestas éstos representan casi un $20 \%$ del total, con un destacado rol de las organizaciones barriales, especialmente en distritos muy densamente poblados como los del área metropolitana de la Ciudad de Buenos Aires.

El 10\% restante de los apoyos registrados corresponde a un conjunto heterogéneo de instituciones que van desde actores corporativos (empresarios y trabajadores) y político 
partidarios, hasta compañías privadas que han prestado apoyo técnico al proceso, pasando por organizaciones de carácter más plural, tales como mesas de concertación locales, que han contribuido a dotar de legitimidad y transparencia a esta política.

Destaca al respecto que desde 2009 funciona la Red Argentina de Presupuestos Participativos (RAPP), integrada por gobiernos locales con PP, la cual cuenta a la fecha con veintidós municipios que se han adherido formalmente a ella como miembros titulares. También pueden integrar la RAPP como miembros adherentes: «Universidades, organizaciones de la sociedad civil, y ciudadanos y ciudadanas comprometidos/as activamente en el desarrollo de experiencias de presupuesto participativo» (art. $3^{\circ}$ del estatuto de la RAPP). A su vez, la RAPP tiene como miembros permanentes a las dependencias del Poder Ejecutivo nacional que han promovido su creación: la Secretaría de Relaciones Parlamentarias de la Jefatura de Gabinete de Ministros y la Secretaría de Asuntos Municipales del Ministerio del Interior.

Como conclusión, se resalta que es clave para extender y mejorar los procesos de PP en Argentina el trabajo en red entre organizaciones gubernamentales de todo nivel y tipo, técnicas y políticas, instituciones académicas y otros actores de la sociedad civil.

\section{LA EXPERIENCIA DE LA UNIVERSIDAD NACIONAL DE GENERAL SARMIENTO EN LA PROMOCIÓN E IMPLEMENTACIÓN DEL PRESUPUESTO PARTICIPATIVO}

Desde el año 2006, la Universidad Nacional de General Sarmiento acompaña y articula con diferentes actores sociales la promoción e implementación de presupuestos participativos en el nivel local de gobierno. En esta sección se desarrolla brevemente el trabajo realizado por la Universidad en relación a esta importante política pública.

Una primera actividad consistió en apoyar a organizaciones sociales y políticas del municipio de San Miguel que impulsaban el desarrollo del PP en el distrito. Posteriormente se acompañó y asistió al propio municipio en la implementación de esta 
política. Asimismo, desde el año 2008 la UNGS asiste al municipio de Zárate en la formulación e implementación del PP en su territorio.

A su vez, la UNGS ha participado, en el marco de la RAPP, en diversas actividades de promoción del PP en diferentes puntos de país, como las desarrolladas en los municipios de La Costa, Tapalqué, Benito Juárez y Cañada de Gómez (pertenecientes a la zona central del país), y en eventos de capacitación y sensibilización a funcionarios locales de los municipios de Benito Juárez (Buenos Aires), Cañada de Gómez (Santa Fe) y Bella Vista (Corrientes), a lo largo del año 2010.

En el caso del desarrollo del PP en el municipio de San Miguel, en un primer momento el aporte más significativo de la Universidad fue su apoyo a la movilización popular y el asesoramiento técnico en materia legislativa, que alentaron la sanción por parte del poder legislativo local de un mecanismo de democracia participativa, en un municipio con una tradición política de oposición a ella. Ya en el año 2006, antes de la promulgación de la ordenanza, se realizó en el campus de la Universidad un congreso de organizaciones sociales que generó articulación entre los actores para insistir nuevamente en el proyecto de ordenanza. Posteriormente, la UNGS jugó un rol clave contribuyendo a que el tema del PP se instalara en buena parte del territorio. Ello fue posible por el desarrollo de un trabajo denominado: Ampliación y sustentabilidad democrática: contribuciones para la implementación de la ordenanza sobre el presupuesto participativo en la localidad de San Miguel. Uno de los principales productos de este proyecto fue la elaboración de una cartilla sobre presupuesto municipal, presupuesto participativo y formulación de proyectos. La misma fue utilizada en doce encuentros de capacitación que se realizaron en tres barrios del distrito a los que asistieron cientos de participantes.

En el año 2008 se comienza a implementar el PP y, a propuesta de la UNGS, el gobierno de San Miguel creó la figura de los «promotores» del PP. Se trata de personas que normalmente no participaban en la estructura formal del municipio antes de la 
adopción del PP y por lo general (pero no necesariamente) son jóvenes estudiantes o graduados del nivel superior de educación (profesorados terciarios o universitarios) que conocen el territorio. Su principal misión consiste en facilitar, moderar y organizar los foros barriales, así como promover y difundir el PP. Para ello son capacitados en temas como finanzas públicas municipales, formulación de proyectos, diagnóstico participativo y comunicación popular, así como en el marco político-normativo e histórico del PP, siendo también recomendable que conozcan a fondo el funcionamiento interno y los servicios prestados por el gobierno municipal para que puedan asesorar mejor a las personas que asisten a las asambleas.

En nuestra experiencia, estos promotores y promotoras del PP lograron en todos los casos llevar adelante con éxito la compleja tarea de romper la barrera de escepticismo que suele presentar la población, especialmente en aquellos distritos con escasa tradición participativa. Ello fue posible por el esfuerzo, la coordinación, el entusiasmo y la formación del equipo y, por el hecho de tratarse de personas que la población no identificaba con los atributos (no siempre exentos de prejuicios) más negativos de la gestión local, como el clientelismo y el paternalismo de los funcionarios políticos o la falta de motivación y excesiva burocratización en lo que concierne al personal técnico. La labor realizada por estos estudiantes fue valorada muy positivamente por el gobierno municipal y sobre todo por los ciudadanos que participaron en el proceso de deliberación: en los diagnósticos participativos, en la formulación de los proyectos y finalmente en la selección de los mismos en asamblea. La experiencia de 50 estudiantes de la UNGS que trabajaron como promotores del PP en el año 2008 continuó con treinta estudiantes en el año 2009 y doce en el 2010.

La experiencia de interacción entre la UNGS y el municipio de Zárate se enmarca en el desarrollo del PP en un distrito de escala mediana y fuerte diversidad territorial. En este caso, la UNGS trabajó con el gobierno municipal desde fines del año 2008. Al igual que en San Miguel, se empezó con una prueba piloto de sensibilización de la población en algunos barrios, labor en la que participó un grupo de estudiantes de la UNGS 
utilizando una cartilla de capacitación elaborada por la propia Universidad. Al mismo tiempo, se asesoró al municipio en el diseño de la reglamentación y las características del PP, y en la programación de su implementación. A comienzos del año 2010 se procedió a la formación de estudiantes de distintos establecimientos de educación superior de la zona de Zárate como promotores del PP a fin de desarrollar la experiencia en todo el territorio del distrito. Tal estrategia ha mostrado ser acertada dado que la labor de dichos promotores y promotoras fue muy positivamente valorada tanto por la población como por los equipos técnicos y políticos del municipio.

Entre las singularidades del municipio de Zárate relacionadas con el PP, se puede mencionar que su cabecera está densamente poblada y que cuenta con una zona rural y una zona insular, presentando esta última alarmantes niveles de atraso relativo respecto al desarrollo del resto del territorio, lo que a priori implica una mayor complejidad en las problemáticas sociales y en la formulación de los proyectos. El grado de participación de los vecinos logrado en el primer año fue relativamente alto comparado con otras experiencias y con las expectativas previas, y la calidad del proceso fue muy positivamente valorada por los participantes. Una prueba de ello es el autoreglamento consensuado al final del primer proceso de PP, donde se formalizó un aspecto que ya había surgido espontáneamente en algunos foros y que mostraba el nivel de conciencia cívica y comunitaria logrado por el PP: la adopción de criterios de justicia social en la distribución territorial de los fondos. En este municipio se prevé que la UNGS pueda aún realizar aportes valiosos, por ejemplo en temas como el asesoramiento técnico para definir criterios, la sensibilización al momento de seleccionar los proyectos, o la evaluación de un proceso notable por su calidad.

Finalmente, la UNGS acaba de convertirse en la primera Universidad aceptada por la RAPP como miembro adherente, consolidando así su compromiso institucional con la promoción del presupuesto participativo.

A partir del análisis de las actividades reseñadas, puede apreciarse el potencial de 
recursos que las instituciones académicas, como la UNGS, puede poner en juego en beneficio de la promoción, formulación e implementación de la política de PP, tanto en términos de asistencia técnica como en roles menos tradicionales, al instituirse como verdaderos actores sociales con capacidad para influir y articular propuestas superadoras en pos de fortalecer la democracia en nuestros países.

\section{CONCLUSIONES}

El concepto de gobernanza, según su tratamiento en el presente artículo, resulta útil para reflejar la relación entre los cambios en el funcionamiento de las administraciones públicas locales, inspirados en parte por el paradigma de la nueva función pública, y la promoción del desarrollo local, entendido éste como fenómeno polisémico que incluye, no exhaustivamente, la actividad económica, las condiciones de vida, la cuestión medioambiental y la profundización de la vida democrática. En relación con este último objetivo, y enmarcándolo en el concepto de gobernanza, el PP puede realizar una relevante contribución.

En Argentina, la aplicación del PP ha nacido al calor de la peor crisis de representación del período posdictatorial y su desarrollo en el país muestra el potencial del que, a nuestro entender, es uno de sus objetivos fundamentales, expandir la experiencia democrática, complementando la democracia delegativa con la participativa y aumentando paralelamente la legitimidad del sistema representativo de gobierno.

A nivel general, el PP, que surgió a comienzos del milenio en Argentina, se encuentra desde el año 2008 en su etapa de mayor expansión histórica, implementándose actualmente en 41 gobiernos locales que abarcan a un cuarto de la población del país, es decir, a unos once millones de personas.

Pero tal difusión tiene un sesgo regional dado que los municipios que cuentan con PP se concentran especialmente en el centro del país y en menor medida en el sur, es decir, en las zonas más desarrolladas, y tiene mucho menor arraigo en el norte, y especialmente 
en el noroeste donde sólo un municipio lo aplica.

En cuanto al tratamiento jurídico y normativo que recibe el PP en Argentina, el marco constitucional y los tratados internacionales con rango constitucional refrendados por la nación contemplan como derecho la participación popular en los asuntos de gobierno y establecen algunos mecanismos de democracia directa como complementarios al carácter representativo de la democracia consagrada como forma de gobierno.

En ese marco, una serie de leyes, decretos y ordenanzas, provinciales y municipales, fomentan o consagran la aplicación del PP. Sin embargo, este tipo de antecedentes, formalmente valiosos, no implican siempre la adopción del PP por parte de los territorios en cuestión. Así, el programa del Poder Ejecutivo nacional Promoción y Apoyo a la Implementación del Presupuesto Participativo ha tenido un alto impacto sobre el incremento en el número de municipios con PP en Argentina.

El PP, en tanto presupuesto, consiste en distribuir parte del gasto público local, cuyo objeto específico se somete al debate de la población. En Argentina el reparto del monto total del PP es predominantemente territorial, sin considerar una base temática, más allá de las experiencias de PP joven que varios municipios vienen desarrollando. A su vez, la forma en que se distribuyen territorialmente los recursos suele ser una potestad del gobierno municipal, al menos al inicio de los distintos procesos de PP. Una vez madurada la experiencia, en varios casos tal distribución se ha modificado en el marco de los reglamentos dictados por los propios participantes. El paso usual de un criterio de reparto igualitario a otro que considere cuestiones de justicia social, por decisión estatal o popular, marca claramente el potencial redistributivo y reequilibrador del PP.

En cuanto al PP como política de participación popular, las diversas formas en que los gobiernos locales conciben y contabilizan la participación impiden una comparación directa, aunque puede afirmarse que una estrategia comunicacional adecuada y la ejecución en tiempo y forma de los proyectos que surgen de los foros son condiciones 
necesarias para lograr y mantener niveles de participación significativos.

Cualitativamente, para que la participación sea tal es requisito contar con procesos previos de información a la población, y la calidad de tal participación dependerá en buena medida de los niveles de involucramiento previstos para la ciudadanía. Así, diagnosticar es mejor que sólo elegir alternativas, diseñar proyectos supera al mero diagnóstico, y el control ciudadano de los proyectos enriquece aún más la participación.

Respecto a los cambios que implica para la gestión local, el PP facilita los procesos de descentralización municipal, colabora en la instalación de una cultura de control y de un modelo de gestión participativa con foco en la producción de valor público al poner cara a cara a los funcionarios y a la población, a la vez que, a nivel político, muestra las potencialidades de compartir el poder para ganar legitimidad.

Desde el punto de vista de la gestión participativa y, en definitiva, de la gobernanza, el PP, en tanto política pública, evidencia la necesidad de contar con el apoyo y la movilización de recursos diversos por parte de un grupo complejo de actores que excede a las autoridades locales, incluyendo a otros niveles de gobierno, al sector privado, a organizaciones de la sociedad civil y a las instituciones académicas. 


\section{BIBLIOGRAFÍA}

Aguilar Villanueva, Luís, 1996, «Estudio introductorio», en Problemas públicos y agenda de gobierno, compilado por Aguilar Villanueva, Luís, Miguel Ángel Porrúa, México.

Blanco, Ismael y Ricard Gomà, 2003, «Gobiernos locales y redes participativas: retos e innovaciones», en Reforma y Democracia. Revista del CLAD, n. 26. CLAD, Caracas.

Boisier, Sergio, 1999, «Desarrollo (local): ¿de qué estamos hablando?», en Revista Paraguaya de Sociología, n. 104, Centro Paraguayo de Estudios Sociológicos, Asunción, pp. 7-30.

Cabannes, Yves, 2005. Presupuesto participativo y finanzas locales. Documento base, red URBAN, n. 9, Alcaldía Porto Alegre, Porto Alegre.

—_, 2004, 72 Frequently Asked Questions About Participatory Budgeting, UNHABITAT, Quito.

Caplán de Cohen, Norma, 2000, «La clave de las finanzas municipales», en Revista Enoikos, n. 16, Facultad de Ciencias Económicas de la Universidad de Buenos Aires, Buenos Aires, pp. 65-76.

Genro, Tarso y Ubiratan Souza, 1998, Orçamento participativo. A experiencia de Porto Alegre, Fundaçáo Perseu Abramo, Porto Alegre.

Gómez Castañeda, Juan, 1995, «Técnicas de elaboración presupuestaria», en Documentos de Trabajo Serie Gobierno y Administración Pública, n. 195, Instituto Universitario de Investigación Ortega y Gasset, Madrid.

Kerz, Mercedes y Santiago Leiras, 2004, «Que veinte años no es nada... Algunas 
reflexiones en torno a los difíciles tiempos de la democracia argentina», en Revista SAAP, vol. 1, n. 3, Sociedad Argentina de Análisis Político, Buenos Aires, pp. 475-488.

López, Andrea, 2003, «La nueva gestión pública: algunas precisiones para su abordaje conceptual», en Serie I: Desarrollo Institucional y Reforma del Estado, Documento n. 68, Dirección de Estudios e Información del Instituto Nacional de la Administración Pública, Buenos Aires.

Mayntz, Renate, 2001, «El Estado y la sociedad civil en la gobernanza moderna», en Reforma y Democracia, Revista del CLAD, n. 21. CLAD, Caracas.

— , 2000, «Los nuevos desafíos de la teoría de la "governance”», en Instituciones y Desarrollo, n. 7, noviembre, Instituto Internacional de Gobernabilidad, Madrid.

Moore, Mark, 1998, Gestión Estratégica y creación de valor en el Sector Público, Paidós, Buenos Aires.

Natera, Antonio, 2004, La noción de gobernanza como gestión pública participativa y reticular, Documento de trabajo «política y gestión», Universidad Carlos III, Madrid.

Navarro Gómz, Carlos, 2002 «Gobernanza en el ámbito local». Ponencia VII Congreso del CLAD sobre Reforma del Estado y de la Administración Pública, Lisboa.

O’Donnell, Guillermo, 2004, «Accountability horizontal: la institucionalización legal de la desconfianza política», en Revista Española de Ciencia Política, n. 11, Asociación Española de Ciencia Política y de la Administración, Madrid, pp. 11-31.

Pierre, Jon, 2000, Debating Governance. Oxford University Press, Oxford.

Pierre, Jon y Guy Peters, 2000, Governance, Politics and the State, MacMillan Press, 
Londres.

PNUD, 2002, Informe sobre desarrollo humano 2002: profundizar la democracia en un mundo fragmentado, Programa de Naciones Unidas para el Desarrollo, New York.

Santos, Boaventura de Sousa, 2004, Democracia y participación: el presupuesto participativo de Porto Alegre, Abya-Yala, Quito,

Texeira, Ana, Grazia de Grazia, Maria Albuquerque y Pedro Puntual, 2004, «Presupuesto participativo. Democratización de la gestión pública y control social», en Fórun Nacional de Participacao Popular, Porto Alegre.

\section{NOTAS}

1 Las distintas definiciones del concepto de desarrollo local, dentro de las diferentes formas de adjetivación del desarrollo, se presentan en Boisier (1999).

${ }^{2}$ Para un excelente estado del arte acerca de las formas en que se configura la agenda pública, en el marco amplio de los estudios sobre el ciclo de las políticas públicas, ver Aguilar Villanueva (1996).

${ }^{3}$ Es clara la relación entre esta nueva gestión local y la denominada nueva gestión pública, en su versión posliberal. Los cambios en dicho concepto, presentes en el paulatino reemplazo de las tres «e» (economía, eficiencia y eficacia) por un arco más amplio de principios, que incluyen la adaptabilidad, innovación, representación y participación como guías para la acción estatal, pueden consultarse en López (2003).

4 Se puede relacionar la expansión del campo de acción de los gobiernos locales, en términos de complejidad y diversidad de sus tareas, con el efecto concomitante de la descentralización, modernización, integración, apertura, urbanización y agudización de la pobreza urbana, de gran vigencia en la actualidad. Caplán de Cohen (2000).

${ }^{5}$ Para un debate profundo sobre el concepto de gobernanza y los actores implicados en ella ver Mayntz (2000), Mayntz (2001), Pierre y Peters (2000) y Pierre (2000).

${ }^{6}$ «En síntesis, tanto la democracia como el republicanismo tienden hacia el polo de una alta decisividad, o efectividad, del poder político [...] En cambio, el liberalismo tiende al polo de la protección, centrado en la esfera privada» (O’Donnell 2004:16).

${ }^{7}$ Los efectos sobre la participación de la ciudadanía se detallan en Cabannes (2005:43), Cabannes (2004:6) y Teixeira et al. (2004:5). Los resultados en términos de discriminación positiva y atención a grupos vulnerables y de erosión del clientelismo político se estudian en Cabannes (2005:43) y Teixeira et al. (2004:5). Las potencialidades en materia de mejoramiento de la gestión municipal y control fiscal ciudadano se exploran en Teixeiraet al. (2004:5). La riqueza de combinar la democracia directa y la representativa se describe en Genro y Souza (1997:12). Finalmente, el logro de relaciones más democráticas entre Estado y sociedad en el seno de ámbitos públicos no estatales, es investigado en Teixeira et al. (2004:5), y Genro y De Souza (1997:12).

${ }^{8}$ La técnica de presupuesto base cero se analiza en Gómez (1995:33-45).

${ }^{9}$ La ciudadanía fiscal juega, con otras cuestiones, un rol importante en el proceso de democratización de la democracia, tal como se detalla en Kerz y Leiras (2004:485).

${ }^{10}$ El concepto de valor público se desarrolla extensamente en Moore (1998:59-100). 
Fecha de recepción: 15 de abril de 2011.

Fecha de aceptación: 15 de agosto de 2011. 